\title{
Online Energy Management System for Distributed Generators in a Grid-Connected Microgrid
}

\author{
Adriana C. Luna*, Nelson L. Diaz ${ }^{* \dagger}$, Moisès Graells ${ }^{\ddagger}$, Juan C. Vasquez*, and Josep M. Guerrero* \\ *Dept. Energy Technology, Aalborg University, Aalborg, Denmark \\ ${ }^{\dagger}$ Dept. Electronic Engineering, Universidad Distrital, Bogota, Colombia,

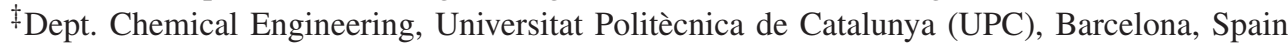 \\ Email: acl@et.aau.dk,nda@et.aau.dk \\ http://www.microgrids.et.aau.dk
}

\begin{abstract}
A microgrid is an energy subsystem composed of generation units, energy storage, and loads that requires power management in order to supply the load properly according to defined objectives. This paper proposes an online energy management system for a storage based grid-connected microgrid that feeds a critical load. The optimization problem aims to minimize the operating cost while maximizing the power provided by the renewable energy sources. The power references for the distributed energy resources (DER) are scheduled by using CPLEX solver which uses as input current measurements, stored data and adjusted weather forecast data previously scaled in each iteration considering the current status. The proposed structure is tested in a real time simulation platform (DSPACE 1006) for the microgrid model, by using Labview to data acquisition and Matlab to implement the energy management system. The results show the effectiveness of the proposed energy management system for different initial conditions of the storage system.
\end{abstract}

\section{INTRODUCTION}

A microgrid (MG) is an energy system consisting of distributed generators, energy storage systems (ESS) and loads, that can operate interconnected to the main grid or in islanded mode [1]. However, when there are several available energy resources to supply the demand, the energy supplied by each one should be scheduled to get an optimal dispatch regarding specific objectives such as economical, technical and environmental aspects [2], [3]. Particularly, storage-based microgrids can provide economic benefits without the need of shifting or shedding loads with the inclusion of a energy management system (EMS) [4] which is essential for critical loads that must be uninterruptedly fed [2].

Regarding economical issues, the main objective is to minimize the operating cost by scheduling the dispatchable units [3]. Another issue in the optimization process is related to make full use of renewable energy sources (RES) because of their intermittent nature as well as to prolong the lifetime of the ESS [5]. Therefore, having well-sized ESS it is possible to enssure that the power supplied by RESs during the high generation periods will be available when the load requires it [6]. For instance, [7] and [8] present energy management systems performed to maximize power generation of hybrid active power generators in grid-connected microgrids based on wind turbine (WT) generator (WT+ESS) and a photovoltaic (PV) generator $(\mathrm{PV}+\mathrm{ESS})$ respectively.
Moreover, hierarchical control is structured to deal with the behavior of the microgrids at different bandwidth [9]. The highest control levels deal with optimal operation and power flow management in a low bandwidth giving the references to the lowest level controller. Whereas, the lowest control levels are responsible of power quality control and regulating local variables in a higher bandwidth in order to assure a good performance of the whole system [15]. In [16] a Constant Power Generation (CPG) for PV systems is implemented where a certain percentage of the energy is cut off arbitrarily in a long-term operation when the output power reaches a certain level. Optimization techniques have been researched as linear and nonlinear approaches [3], even when these methods are often used offline. Recently, some online energy management system for microgrids (MG-EMS) have been implemented as in [4], but it does not considered safety ranges for the operation of the ESS which ensures longer ESS lifetime [12].

In this paper, an online strategy of EMS is developed by considering operating costs, aimed to reduce the energy consumption from the main grid power and maximize the use of RESs in order to supply permanently a constant load as well as hold the state of charge (SoC) of the ESS in safety operation ranges. The proposed EMS is tested in a MG composed of two RESs (a WT and a PV array), an ESS, and a critical load connected to the main grid (Fig. 1). The power references of RESs and ESS are scheduled while the primary controllers are responsible of managing the operational modes.

\section{OPERATION OF THE MICROGRID}

The MG used as study case in this paper consists in two RESs and an ESS to feed a critical load which always requests a fixed power (Fig. 1). Since the MG is connected to the main grid, the local controllers of the distributed energy resources (DER), i.e. RESs and ESS, are working in current control mode (CCM) [10].

The RESs primary controllers are either following the power reference given by the MPPT algorithm or the power references derived from the optimization procedure $\left(P_{v}^{*}\right.$ and $P_{w}^{*}$ in Fig. 1). Indeed, the controllers follow the scheduled power just if this is lower than the maximum available power. Particularly, the local controller activates the PControl mode 


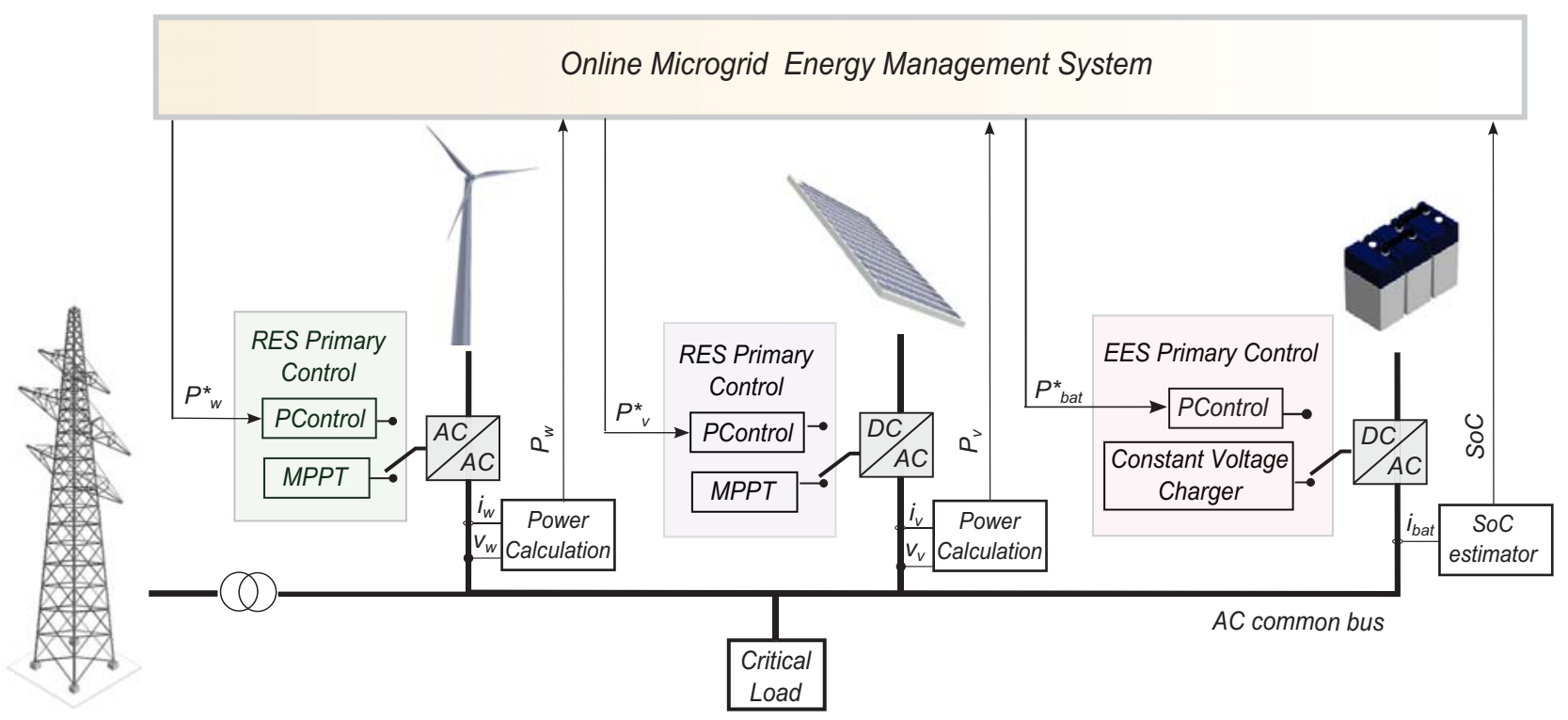

Fig. 1. Scheme of the proposed MG

when battery is charged in order to avoid storage overcharging and limits grid power injection [11].

Furthermore, a very effective way of charging a leadacid battery is by means of a two-stage procedure which involves two different control loops [12], [13], [14]. Typically, charging/discharging mode should be alternated by a constant voltage charge stage when the battery voltage reaches a threshold voltage $V_{r}$. This is particularly critical for ensuring longer lifetime batteries [12]. During the constant voltage charge stage, the battery voltage should be kept constant and consequently, the current will approach to zero asymptotically, and once it falls below a certain value, the battery is considered as fully charged [12], [14]. At this point, the ESS takes as much power as required to keep its battery voltage at $V_{r}$ [13]

In this paper, the battery will be considered as charged when the battery array reaches $V_{r}$ and the local controller moves to constant voltage charge stage. While, when the battery is not charged, the local controller is activated in PControl mode in which the power references profile is given by the online EMS assuring that the ESS is charged or discharged in an optimal way according to economical issues.

On top of that, since the microgrid is grid-connected, the main grid regulates the common bus and thus, it injects/absorbs the required power to support the unbalance between generation, demand and storage in the microgrid.

\section{PROPOSED ENERGY MANAGEMENT MODEL}

\section{A. Required Measurements}

An online MG-EMS sets the power reference profiles for the DERs considering measurements of the MG sampled in a lower bandwidth than the one deployed for the local controllers.

The proposed structure considers the measurement of the $\mathrm{SoC}$ for the ESS (SoC in Fig. 1) and power measurements for RESs $\left(P_{v}\right.$ and $P_{w}$ in Fig. 1$)$. The SoC in a battery array represents a ratio between the current extracted/stored energy and the maximum energy that the ESS is able to store. This value is presented in percentage as a function of the current [13].

Furthermore, the measurements required by the MG-EMS cannot be taken directly, thus additional block are included. On one hand, the block SoC estimator measures the output current of the battery inverter and computes the instantaneous SoC, which is taken by the MG-EMS each sample time. This block is based on the well-known Ampere-hour counting method [10], [12]. On the other hand, the block Power Calculation takes the output current and the output voltage of the inverter for each RES and compute the average power such as in [17].

\section{B. Online Scheme}

The general architecture of the proposed online MG-EMS is represented in Fig. 2 which shows the interaction among the basic blocks of the MG-EMS. It is composed of a scheduling caller, data storage, and an algebraic modeling language (AML) which calls a solver.

The scheduling caller is responsible to collect the measured data, process the input data for scheduling and periodically sends a request for running to the $\mathrm{AML}$ and the solver in order to get new power reference profiles. The $A M L$ is a high-level computer programming language to formulate the mathematical optimization problem in an algebraic notation and translates the problem to be solve by a specific solver. The results of the optimization problem for each iteration are updated in a file that is read by the scheduling caller and the new references are sent to the MG. The data storage is composed of a set of files that can be read/written by the scheduling caller or by the AML. 


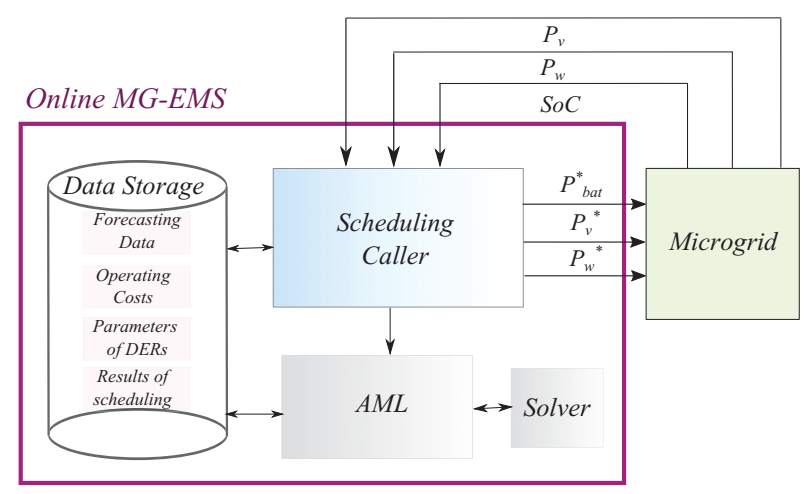

Fig. 2. Structure of the proposed online EMS

The proposed online MG-EMS is performed each $15 \mathrm{~min}$ utes with a time horizon of 4 hours as shown at the bottom of the Output Data block in Fig. 3 in order to handle the variability of the RES.

\section{Scheduling input data processing}

The scheduling caller has the function of processing the input data so that the scheduling can be performed (Fig. 3). The scheduling process requires the MG parameters, operating data, initial condition of the SoC for the ESS and the profiles of the maximum expected power of the RES during the time horizon when the scheduling will be set $\left(P_{g_{\max }}\right.$ in Fig. 3). In the case of the scalar data, the data processing consists in converting the data to a structure that can be read by the AML.

Regarding the maximum expected power profiles of the RES, these are obtained from 24-hour ahead forecasting power profiles which are stored previously as files in the data storage ( $P_{w}$ forecast and $P_{v}$ forecast in Fig. 3 ). These profiles are dynamically adjusted taking into account the current measurement of power, $P_{v}$ and $P_{w}$. The obtained data $\left(P_{w}^{a d j u s t e d}\right.$ and $P_{v}^{\text {adjusted }}$ in Fig. 3) is converted to a structure to be used by the scheduling. The scheduling process produces power reference profiles for the RESs and ESS in the time horizon (4 hours) assuming time slots of 1 hour but this is updated each 15 minutes.

To illustrate the process, in Fig. 3 the forecast power of the WT in the first time slot has a lower value than the measured value, thus the profile of the whole time horizon is adjusted and the result is the $P_{w}{ }^{\text {adjusted }}$ profile. A similar procedure is applied to the profile of the PV in the current time horizon. The results are converted to structure format and they are used as the inputs $P_{g_{\max }}(W T, t)$ and $P_{g_{\max }}(W T, t)$ for the scheduling. The scheduling module sets the power references for the RESs and the battery in an optimal way considering economic issues. In this case, $P_{b a t}{ }^{*}$ is set to a reference different to zero at the first time slot and then the battery is charged and the reference is set to zero for the next three time slots. Meanwhile, the power reference profile of the WT is equal to the available power $\left(P_{w}^{*}=P_{w}^{a d j u s t e d}\right)$ and the power profile of the PV is curtailed at some time slots $\left(P_{v}^{*} \leq P_{v}^{\text {adjusted }}\right)$.
TABLE I

PARAMETERS OF THE MODEL

\begin{tabular}{|c|c|c|}
\hline Name & Description & Value \\
\hline$T$ & Time of scheduling & $4[\mathrm{~h}]$ \\
$\Delta t$ & Duration of interval & $1[\mathrm{~h}]$ \\
$n_{g}$ & Number of power sources & 3 \\
$n_{k}$ & Number of ESS & 1 \\
$P_{L}$ & Critical Load & $600[\mathrm{~W}]$ \\
$P_{\text {losses }}$ & Power losses & $72[\mathrm{~W}]$ \\
$P_{g_{\max }}(i, t)$ & Power max for generators & $0-1.2[\mathrm{~kW}]$ \\
$S O C_{\max }$ & State of Charge max & $100[\%]$ \\
$S O C_{\min }$ & State of Charge min & $50[\%]$ \\
$S O C_{0}$ & Initial Condition & $50-100[\%]$ \\
$\varphi_{\text {bat }}$ & SOC coefficient & $7.5503[\% / \mathrm{kWh}]$ \\
$C(i, t)$ & Coste of generation & {$[0,0,2][\mathrm{DKK} / \mathrm{kWh}]$} \\
$\xi(i)$ & Penalization costs & $2[\mathrm{DKK} / \mathrm{kWh}]$ \\
\hline \multicolumn{2}{|c}{}
\end{tabular}

\section{Optimization Problem}

This problem has been developed as a linear programming (LP) problem with a discrete time representation and the data of each time slot correspond to average values. In order to generate the model, the software GAMS is used as AML and it calls the solver CPLEX.

1) Parameters: The scheduling is performed for $T$ hours in intervals of $\Delta t$ hours with the index $t$ as the elementary unit of time, $t=1,2,3 \ldots T$. The parameters used in this model are presented in table I.

The index $i$ represents the generators, so $i=P V, W T$, grid and the number of power sources is $n_{g}=3$. The input parameters $C(i, t)$ and $P_{g_{\max }}(i, t)$ are sets of real data that correspond to the elementary cost of generation and the maximum power that the $i-t h$ power source can provide. The elementary cost of using the main grid is presented in danish kroners (DKK) and the penalization cost is set as equal to the cost of using the main grid. In this case the elementary costs are set as constant in the time for every generation unit.

$P_{L}(t)$ is the load profile of the critical load. For this particular case study, the load profile is set to $600 \mathrm{~W}$ for all time intervals. $P_{\text {losses }}$ is the power losses set after simulating power load tests in the range of available power.

Regarding the storage, one valve-regulated lead-acid (VRLA) battery is used, thus the number of ESS is $n_{k}=1$. Assuming the average voltage of the storage at $\Delta t$ as its nominal value ( Vbatt $_{n o m}$ ), the current can be represented in terms of power $\left[I_{b a t}(t)=\frac{P_{b a t}(t)}{V_{b a t t_{n o m}}}\right]$, and the SoC can be defined as:

$$
S o C(k, t)=S o C(k, t-1)-\varphi_{b}(k) *\left[P_{b a t}(k, t) \Delta t\right]
$$

where the coefficient $\varphi_{\text {bat }}(k)=\frac{\eta_{c}(k)}{\operatorname{Cap}_{\text {bat }}(k) * \operatorname{Vbatt}_{\text {nom }}(k)}$ is related to the energy capacity and the state of charge of the battery and at $t=1, S O C(k, t-1)$ is replaced by the initial condition $S O C_{0}(k)$. $\operatorname{Vbatt}_{n o m}, \operatorname{Cap}_{\text {bat }}(k)$ and $\eta_{c}(k)$ are nominal values provided by the manufacturer. In this particular case, only one ESS is considered, which is an electric battery whose efficiency $\varphi_{\text {bat }}$ is obtained out of the optimization model. 


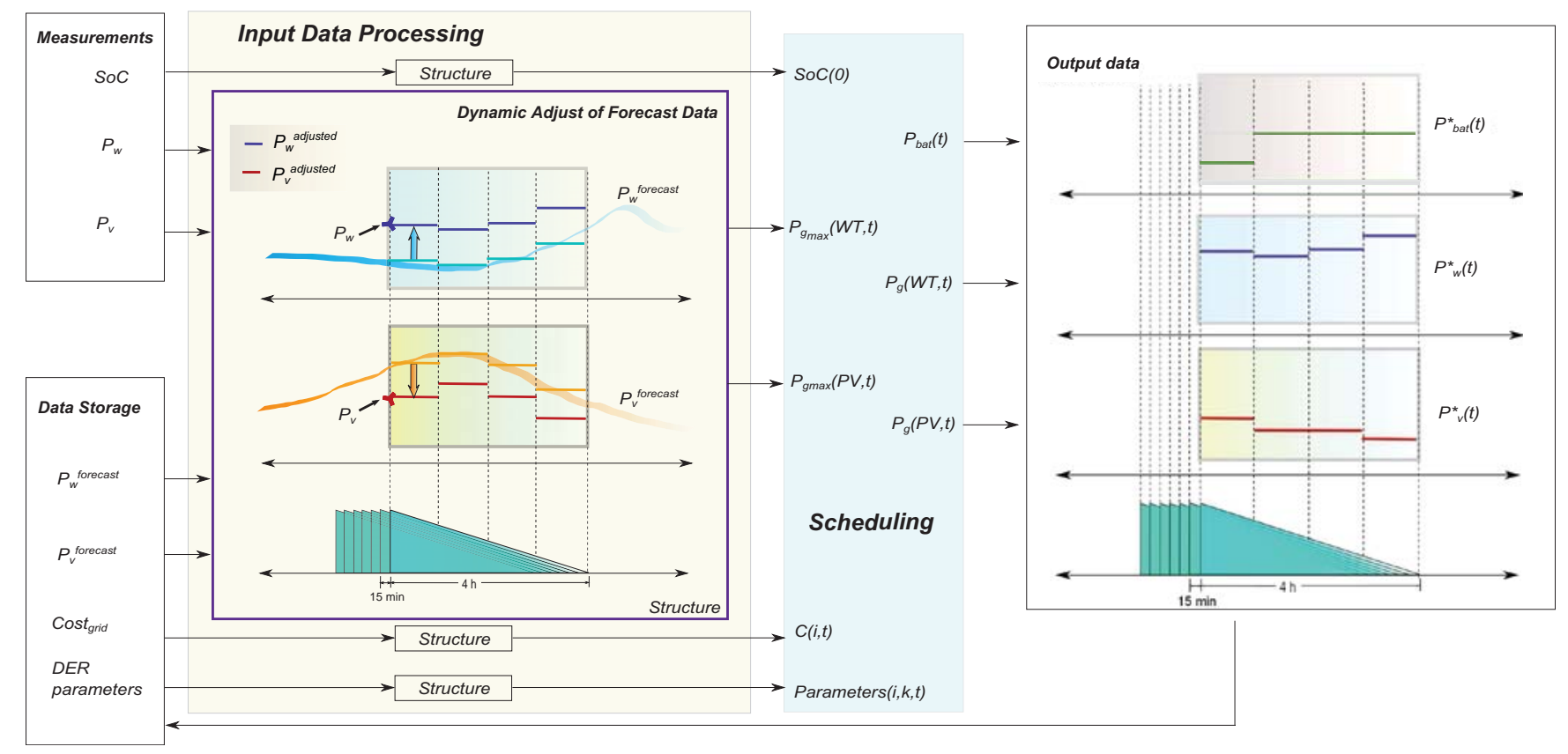

Fig. 3. Description of the data processing.

2) Model: The objective is to minimize the operating cost for which the equation is defined:

$$
\begin{aligned}
\operatorname{COST}= & \sum_{i=1}^{n_{g}} \sum_{t=1}^{T}\left[P_{g}(i, t) \Delta t\right] * C(i, t)+ \\
& \sum_{i=1}^{n_{g}} \sum_{t=1}^{T} \xi(i) *\left[P_{g_{\max }}(i, t) \Delta t-P_{g}(i, t) \Delta t\right]
\end{aligned}
$$

The first term represents the cost that the user has to pay for the electric energy provided by the sources and the second term corresponds to a penalization for performing curtailment to the power of the RES (not using the available energy of the RES at $t$ ). The parameter $\xi(i)$ corresponds to a shadow price.

Additionally, there are some constraints to consider in the model. Firstly, the balance of the energy has to be fulfill:

$$
\begin{array}{r}
\sum_{i=1}^{n_{g}} E g(i, t)+\sum_{k=1}^{n_{s}} E_{\text {bat }}(k, t)= \\
\sum_{l} E_{L}(l, t)+\operatorname{Elosses}(t), \quad \forall t, i, k, l
\end{array}
$$

Wrinting this equation in terms of powers, the balance of energy can ben seen as:

$$
\begin{array}{r}
\sum_{i=1}^{n_{g}} P_{g}(i, t) \Delta t+\sum_{k=1}^{n_{k}} P_{b a t}(k, t) \Delta t= \\
P_{L}(t) \Delta t+\operatorname{Plosses}(t) \Delta, \forall t
\end{array}
$$

Also, the power reference scheduled for the power sources at each $t, P_{g}(i, t)$, must be no greater than the maximum power that can be provided for them at each $t, P_{g_{\max }}(i, t)$.

$$
0 \leq P_{g}(i, t) \leq P_{g_{\max }}(i, t), \forall i, t
$$

Thus, the $S o C(k, t)$ is related to the power in the storage at each $t$ :

$S o C(k, t)=S o C(k, t-1)-\varphi_{\text {bat }}(k) *\left[P_{b a t}(k, t) \Delta t\right], \forall k, t$

Besides, the SoC at each $t$ is bounded. $S o C_{\max }(k)$ is selected to allow the ESS to be fully charged without overcharging and $S o C_{\min }$ is chosen to limit the depth of discharge (DoD) accordingly to the recommendation of the IEEE15612007 standard [12].

$$
\begin{gathered}
S o C_{\min }(k) \leq S o C(k, t) \leq S o C_{\max }(k), \forall k, t \\
\text { IV. RESULTS }
\end{gathered}
$$

\section{A. Hardware in the Loop Results}

The real time simulation of the proposed online MG-EMS is implemented in the AAU Intelligent Lab by using the structure shown in Fig. 4. To perform the real simulation of one day, the data were time-scaled i.e. one hour corresponds to one minute simulation. In the same way, the capacity of the battery and the elementary cost of the grid were scaled.

The microgrid is implemented in dSPACE and the measurements are sent to the computer by using Ethernet communication with UDP protocol. These measurements are read by a program implemented in Labview and some of the data are available to be used for the Scheduling Caller by means of Ethernet communication with TCP protocol. The Scheduling Caller is implemented in Matlab and it is run every $1 / 4 \mathrm{~min}$ which represents the scaled $15 \mathrm{~min}(1 / 4 \mathrm{~h})$.

The online MG-EMS is tested under three different initial conditions of SoC: $80 \%, 100 \%$ and $40 \%$.

The power measurements of the energy resources in the MG in the first case $(S o C(0)=80 \%)$, are presented in Fig. 5. In 


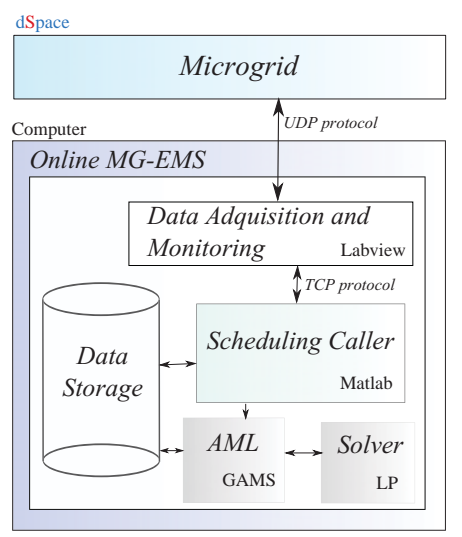

Fig. 4. Structure to performe real time simulation of the proposed online EMS

fact, the results of the RESs include the maximum available power and the measured power, but in this case the two profiles are the same so it is possible to see a difference just in the WT profile at the end of the day (at 1200 s.). Meanwhile, the battery power profile is scheduled to charge/discharge the battery accordingly to the energy balance and as a result the profile of the power absorbed from the main grid is zero most of the time. It can be seen some peaks due to the step nature of the input signals used as maximum values for the RESs.

The process of charging and discharging is presented by means of the SoC in Fig. 6. It is possible to see that the battery is supporting the demand requirements at the beginning and at the end of the day, when the available energy from the RESs is lower that the one required by the load. On the other hand, with this initial condition of SoC, the energy provided by the RES is not enough to consider the battery charged at any time of the day because the voltage of the battery does not reach the threshold voltage $\left(V_{\gamma}=756 \mathrm{~V}\right)$ as shown in Fig. 7 .

Additionally, the hourly and daily accumulated costs are presented in Fig. 8 and 9, respectively. In this case, the costs are due to processing, communication and the nature of the considered signals rather than to the scheduling process. As shown in the first case, the highest cost during the day is obtained when the highest peak appears. Nevertheless, the daily accumulated cost shows that the final cost is 0.072 DKK. The measured and maximum available powers of the energy resources in the MG in the second case $(S o C(0)=100 \%)$, are presented in Fig. 10, whereas the SoC of the battery and its voltage are presented in Fig. 11 and 12, respectively.

As can be seen, the behavior of the whole system is similar to the previous case at the first middle of the day. However, when the battery is charged (Fig. 12), its local controllers change the operational mode to constant voltage charger and the battery does not follow the scheduled profile but as its current tends to zero, the power tends to zero, as well. The power of the main grid presents a peak during the change of the battery operational mode and then, the scheduling of the RES power curtails the available power to assure the energy balance. When the battery voltage cannot be hold equal to the

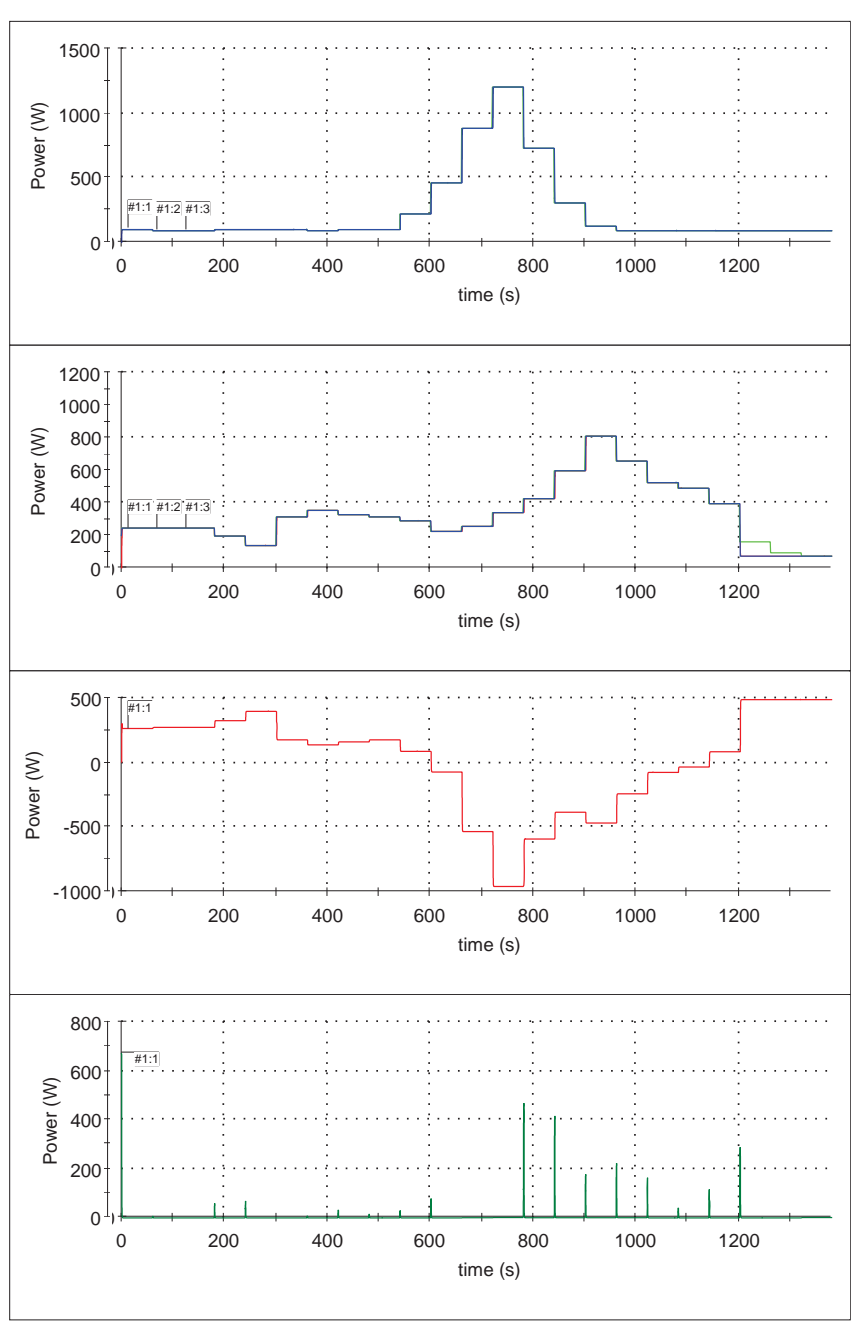

Fig. 5. Power of the devices with $S o C(0)=80 \%$. Top to down: Maximum available and measured power of PV, Maximum available and measured power of WT, Power of battery, Power of the main grid

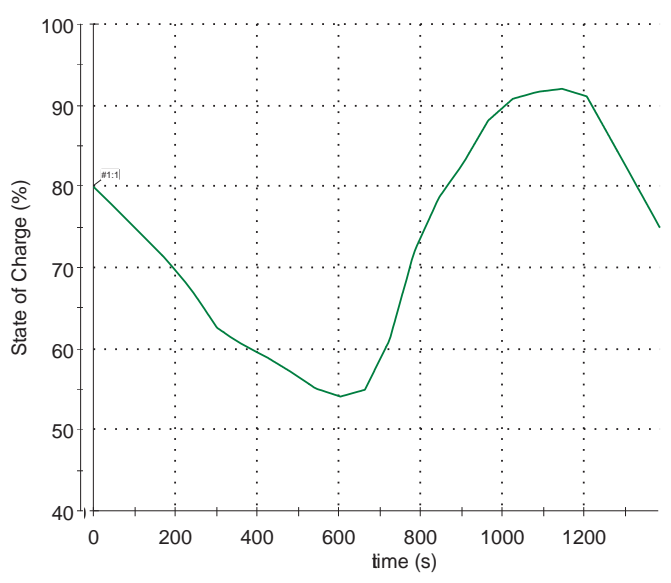

Fig. 6. State of charge with $\operatorname{SoC}(0)=80 \%$ 


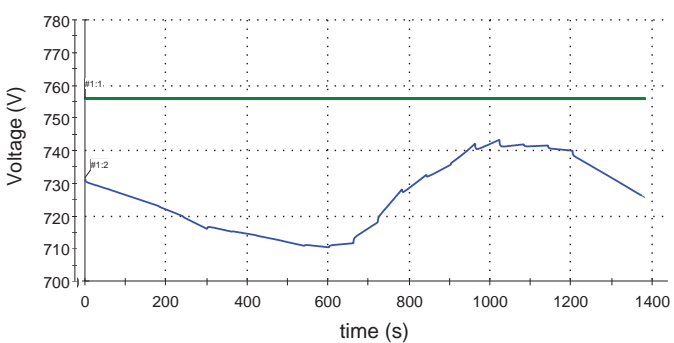

Fig. 7. Battery voltage with $\operatorname{SoC}(0)=80 \%$

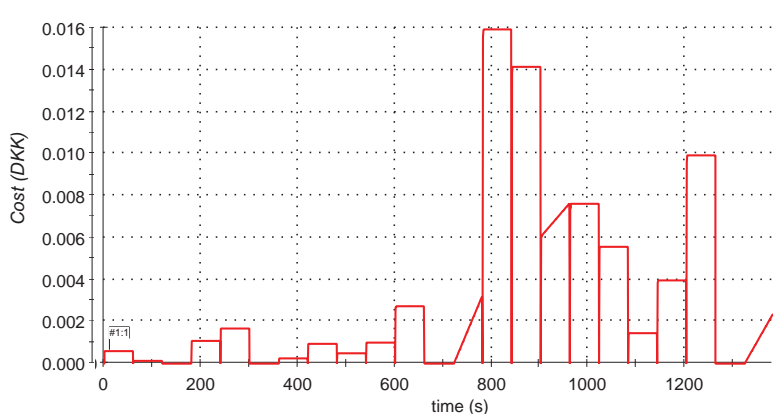

Fig. 8. Hourly accumulative cost of using the grid with $S o C(0)=80 \%$

threshold value, the local controllers of the battery change and follow the scheduled reference again.

Besides, the hourly and daily accumulated costs under the condition $S o C(0)=100 \%$ are presented in Fig. 13 and 14 , respectively. As can be seen in the hourly accumulated cost, the highest cost during the day is obtained when there are changes of operational modes of the battery because the grid has to inject power to regulate those changes. Additionally, in this test some power was required to start the system. Consequently, the daily accumulated cost presents the final cost at the end of the day of 0.17 DKK.

Finally, the ability of the online MG-EMS to recover the system from an initial condition of the $S o C$ below the minimum requirement $(S o C=50 \%)$ is tested by setting $\operatorname{SoC}(0)=40 \%$. The measured and maximum available powers of the energy resources in the MG are presented in Fig. 15, whereas the SoC of the battery and its voltage are presented in Fig. 16 and 17, respectively.

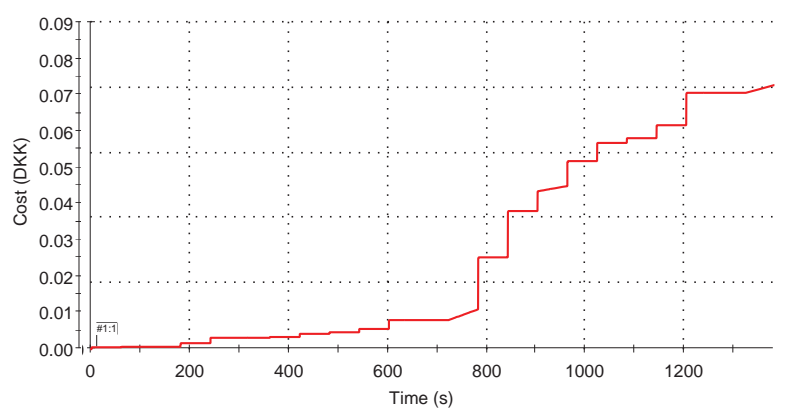

Fig. 9. Daily accumulative cost of using the grid with $\operatorname{SoC}(0)=80 \%$

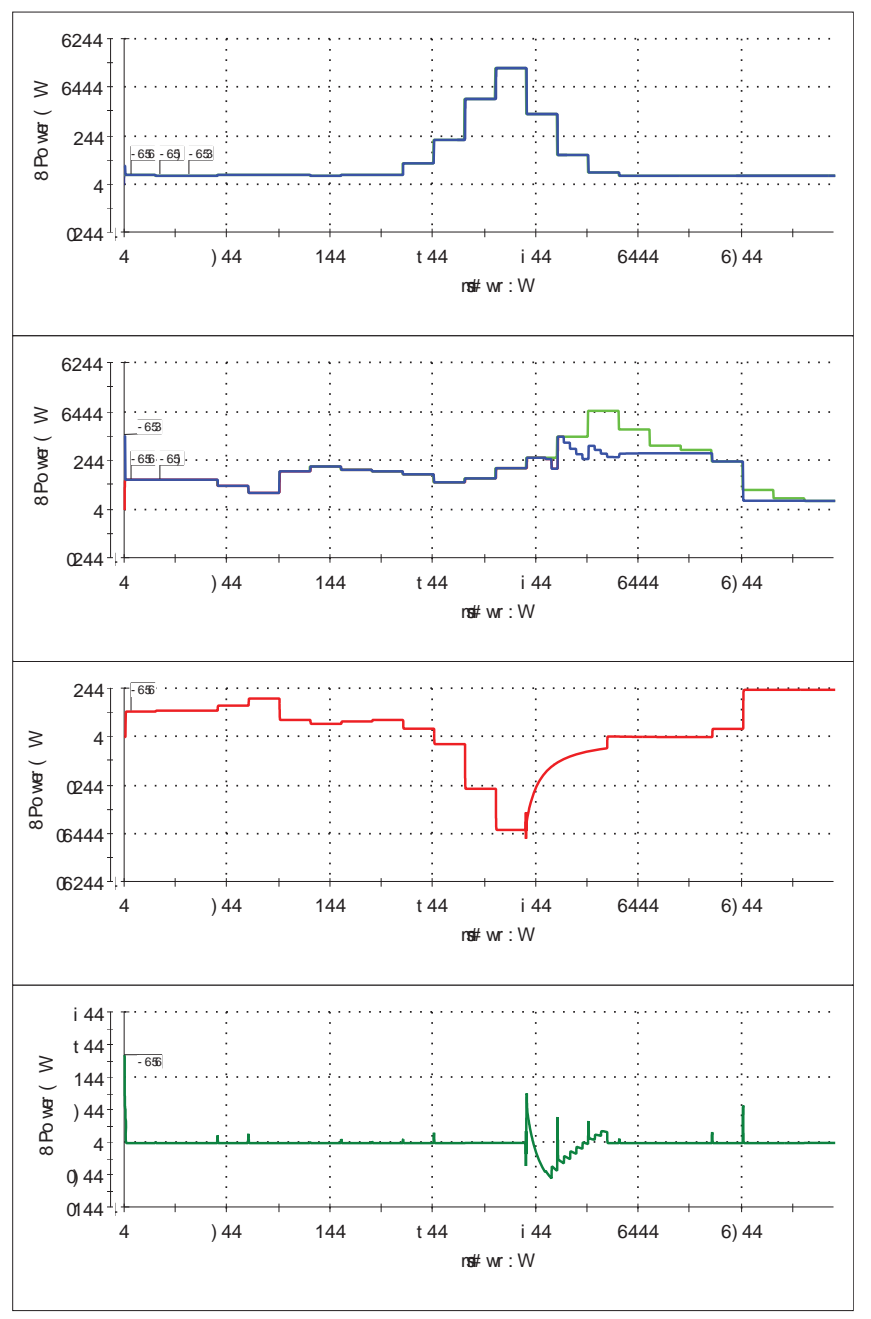

Fig. 10. Power of the devices with $S o C(0)=100 \%$. Top to down: Maximum available and measured power of PV, Maximum available and measured power of WT, Power of battery, Power of the main grid

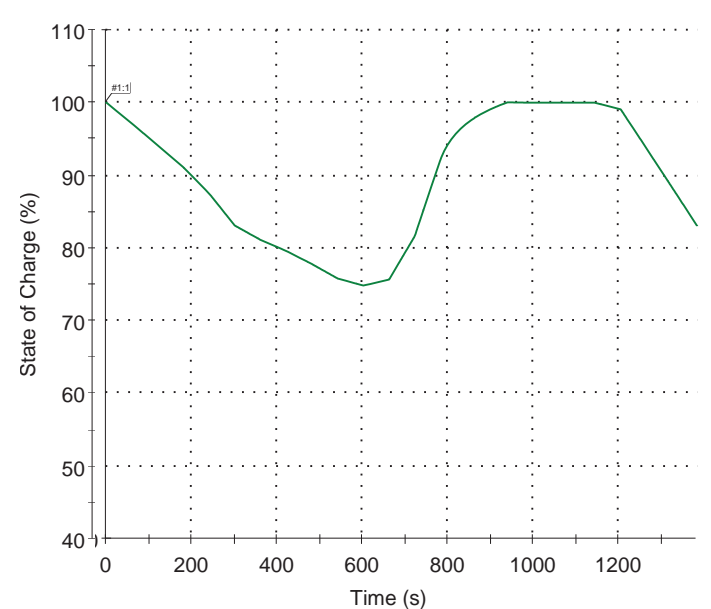

Fig. 11. State of charge with $\operatorname{SoC}(0)=100 \%$ 


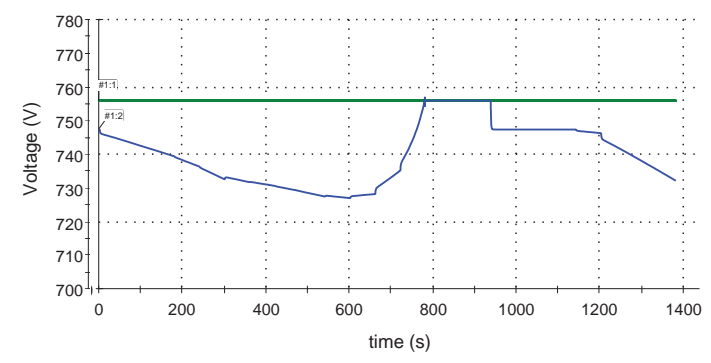

Fig. 12. Battery voltage with $\operatorname{SoC}(0)=100 \%$

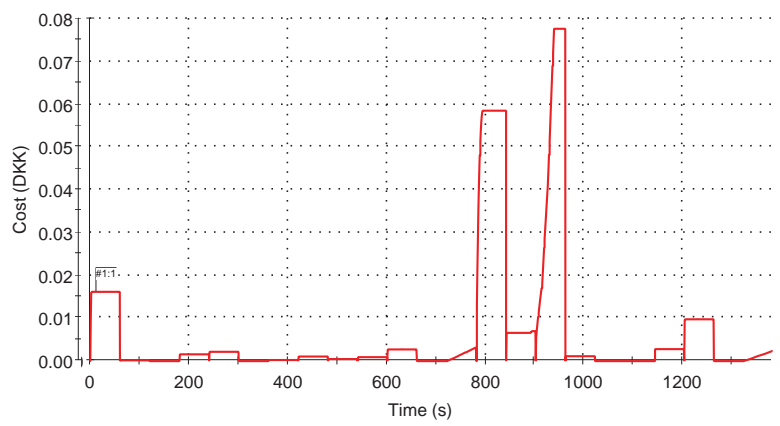

Fig. 13. Hourly accumulative cost of using power from the main grid with SoC $(0)=100$

As expected, the power provided by the RESs is not enough to fully charged the battery at any time of the day (Fig. 17) and therefore, the power profiles of the RESs are not curtailed (Fig. 15), as in the first case $(\operatorname{SoC}(0)=80 \%)$.

Moreover, at the beginning of the day, the main grid provides the energy required by the demand that cannot be provided by the RES but also the energy needed by the battery to be charged until a safe value in a smooth way (Fig. 15 and 16). After that, the power of the battery is hold in zero and the main grid support the unbalance between the demand and the generation provided by the RESs (from 200s to 600s in the battery power profile in Fig. 15). When the power from the RESs are higher than the load, the excedent of energy is stored in the battery and after that, the power absorbed from the main grid is about zero.

The hourly and daily accumulated costs under the condition

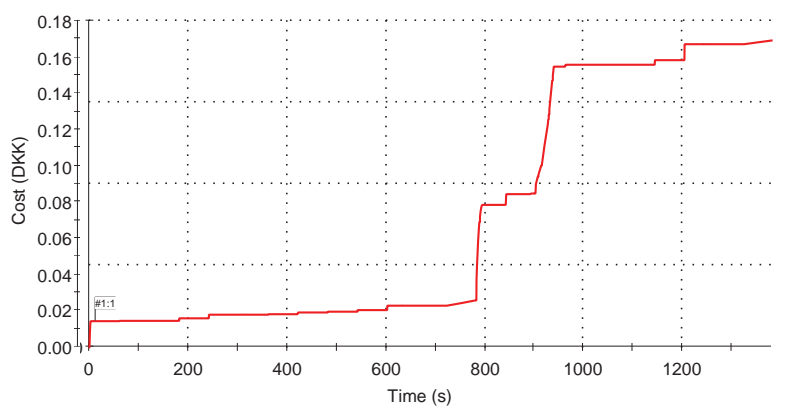

Fig. 14. Daily accumulative cost of using power from the main grid with $\operatorname{SoC}(0)=100$

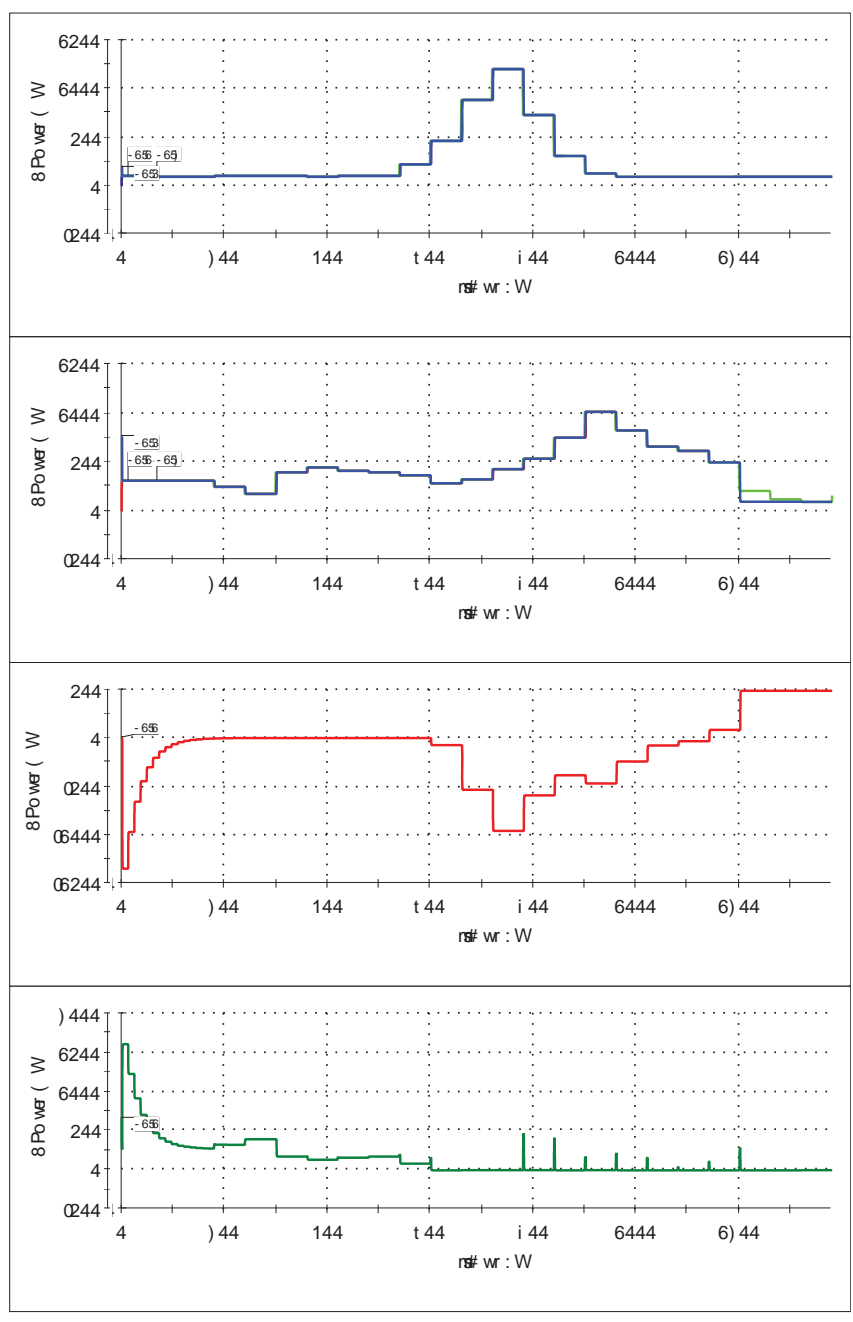

Fig. 15. Power of the devices with $S o C(0)=40 \%$. Top to down: Maximum available and measured power of PV, Maximum available and measured power of WT, Power of battery, Power of the main grid

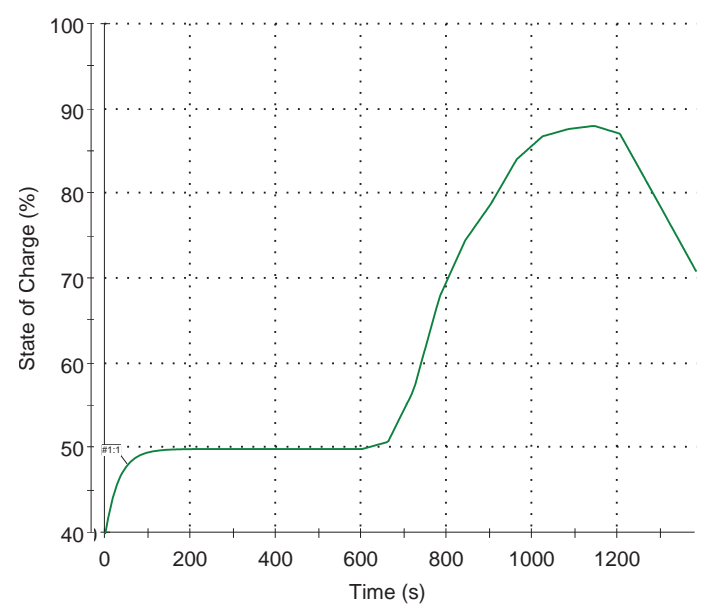

Fig. 16. State of charge with $\operatorname{SoC}(0)=40 \%$ 


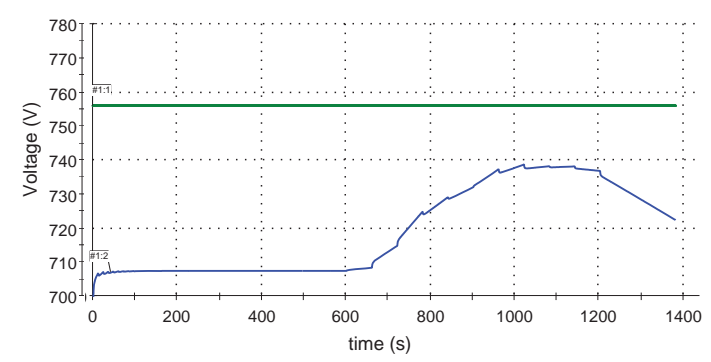

Fig. 17. Battery voltage with $S o C(0)=40 \%$

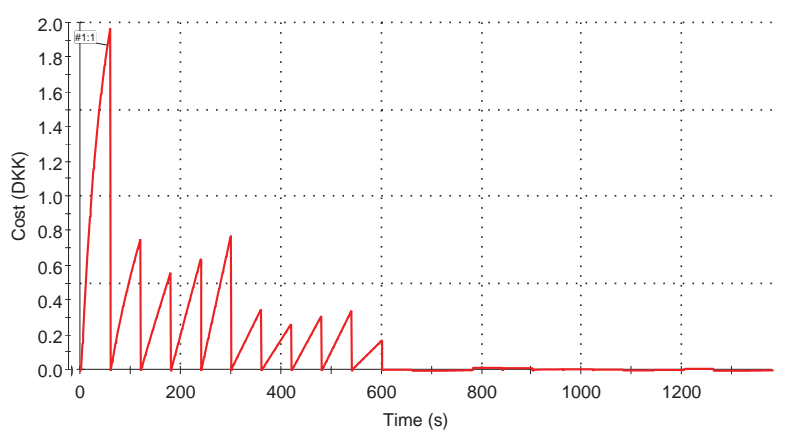

Fig. 18. Hourly accumulative cost of using the grid with $\operatorname{SoC}(0)=40$

$S o C(0)=40 \%$ are presented in Fig. 18 and 19, respectively. As can be seen in the hourly accumulated cost, the highest costs during the day are obtained to recover the battery to safety conditions and after that, to support the demand while holding the battery in the minimum safety condition. In this case, the cost owing to communication is negligible. Consequently, the daily accumulated cost shows as final cost at the end of the day $6.5 \mathrm{DKK}$.

\section{CONCLUSION AND FUTURE WORK}

The proposed online MG-EMS achieves a good commitment between operating costs and the performance of the ESS while assuring the maximum use of the RES. Despite the granularity of the optimization model, the scheduling is economic optimal and the performance of the system is smooth because of the online structure. This approach is performed online each $15 \mathrm{~min}$ with a time horizon of 4 hour holding

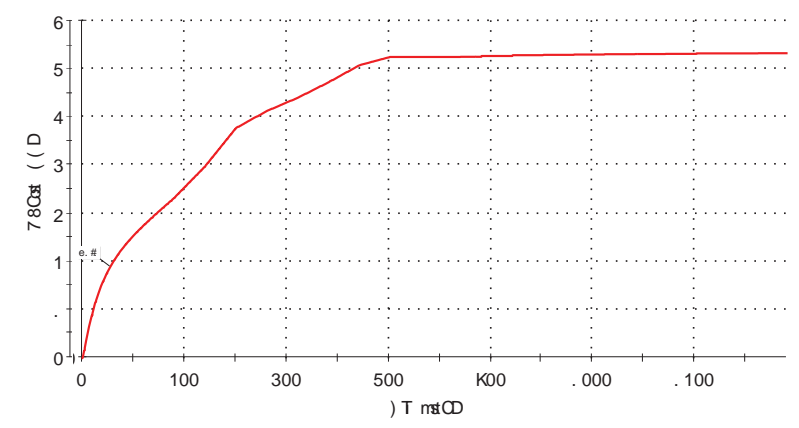

Fig. 19. Daily accumulative cost of using the grid with $\operatorname{SoC}(0)=40$ the battery in safety values of the SoC or even recovering from lower conditions. Besides, including a penalization to the power curtailment of the RES guarantees that the excess of energy is stored in the ESS. As a future work, the operation modes of the ESS can be scheduled in order to improve the results and the system can be enhance with generation units with different features such as low variability.

\section{REFERENCES}

[1] Q. Jiang, M. Xue and G. Guangchao, "'Energy Management of Microgrid in Grid-Connected and Stand-Alone Modes",, IEEE Transactions on Power Systems, Vol. 28, nr. 3, pp. 3380-3389, 2013.

[2] de Matos, J.; e Silva, F.; Ribeiro, L., "Power Control in AC Isolated Microgrids with Renewable Energy Sources and Energy Storage Systems," IEEE Transactions on Industrial Electronics, vol.PP, no.99, pp.1,1.

[3] M. Iqbal, M. Azam, M. Naeem, A.S. Khwaja, A. Anpalagan, Optimization classification, algorithms and tools for renewable energy: A review, Renewable and Sustainable Energy Reviews, Volume 39, November 2014, Pages 640-654

[4] P. Malysz, S. Sirouspour and A. Emadi, "An optimal energy storage control strategy for grid-conneected microgrids," IEEE Transactions on Smart Grid, vol. 5, pp. 1785-1796, July 2014.

[5] Chengshan Wang; Mengxuan Liu; Li Guo, "Cooperative operation and optimal design for islanded microgrid," Innovative Smart Grid Technologies (ISGT), 2012 IEEE PES, pp.1,8, 16-20 Jan. 2012

[6] Marra, F.; Guangya Yang; Traeholt, C.; Ostergaard, J.; Larsen, E., ”A Decentralized Storage Strategy for Residential Feeders With Photovoltaics," Smart Grid, IEEE Transactions on , vol.5, no.2, pp.974,981, March 2014

[7] Kanchev, H.; Di Lu; Colas, F.; Lazarov, V.; Francois, B., ”Energy Management and Operational Planning of a Microgrid With a PV-Based Active Generator for Smart Grid Applications," IEEE Transactions on Industrial Electronics, vol.58, no.10, pp.4583,4592, Oct. 2011

[8] Kanchev, H.; Lazarov, V.; Francois, B., "Environmental and economical optimization of microgrid long term operational planning including PV-based active generators," Power Electronics and Motion Control Conference (EPE/PEMC), 2012 15th International, pp.LS4b-2.1-1,LS4b2.1-8, 4-6 Sept. 2012

[9] Vasquez, J.C.; Guerrero, J.M.; Miret, J.; Castilla, M.; de Vicua, L.G., "Hierarchical Control of Intelligent Microgrids," Industrial Electronics Magazine, IEEE , vol.4, no.4, pp.23,29, Dec. 2010

[10] Wu, D.; Tang, F.; Dragicevic, T.; Vasquez, J.C.; Guerrero, J.M., ”Autonomous Active Power Control for Islanded AC Microgrids With Photovoltaic Generation and Energy Storage System," IEEE Transactions on Energy Conversion, vol.29, no.4, pp.882,892, Dec. 2014

[11] Manuela Sechilariu, Bao Chao Wang, Fabrice Locment, Antoine Jouglet, DC microgrid power flow optimization by multi-layer supervision control. Design and experimental validation, Energy Conversion and Management, Volume 82, June 2014, Pages 1-10, ISSN 0196-8904, http://dx.doi.org/10.1016/j.enconman.2014.03.010.

[12] IEEE Guide for Optimizing the Performance and Life of Lead-Acid Batteries in Remote Hybrid Power System, IEEE Std. 1561- 2007, April 2008.

[13] Dragicevic, T.; Guerrero, J.M.; Vasquez, J.C.; Skrlec, D., "Supervisory Control of an Adaptive-Droop Regulated DC Microgrid With Battery Management Capability," IEEE Transactions on Power Electronics, vol.29, no.2, pp.695,706, Feb. 2014

[14] D. Linden and T. Reddy, Handbook of batteries. McGraw-Hill handbooks, McGraw-Hill, 2002.

[15] Guerrero, J.M.; Vásquez, J.C.; Matas, J.; de Vicuña, L.G.; Castilla, M., "'Hierarchical Control of Droop-Controlled AC and DC MicrogridsA General Approach Toward Standardization,", IEEE Transactions on Industrial Electronics, vol.58, no.1, pp.158,172, Jan. 2011.

[16] Yongheng Yang; Blaabjerg, F.; Huai Wang, "'Constant power generation of photovoltaic systems considering the distributed grid capacity,", Applied Power Electronics Conference and Exposition (APEC), 2014 Twenty-Ninth Annual IEEE, vol., no., pp.379,385, 16-20 March 2014

[17] Coelho, E.A.A.; Cortizo, P.C.; Garcia, P.F.D., "Small-signal stability for parallel-connected inverters in stand-alone AC supply systems," IEEE Transactions on Industry Applications, vol.38, no.2, pp.533,542, Mar/Apr 2002 